\title{
109 new Galactic open clusters ${ }^{\star \star \star}$
}

\author{
N. V. Kharchenko ${ }^{1,2,3}$, A. E. Piskunov ${ }^{1,2,4}$, S. Röser ${ }^{2}$, E. Schilbach ${ }^{2}$, and R.-D. Scholz ${ }^{1}$ \\ 1 Astrophysikalisches Institut Potsdam, An der Sternwarte 16, 14482 Potsdam, Germany \\ e-mail: [nkharchenko; apiskunov;rdscholz]@aip.de \\ 2 Astronomisches Rechen-Institut, Mönchhofstraße 12-14, 69120 Heidelberg, Germany \\ e-mail: [nkhar;piskunov; roeser;elena]@ari.uni-heidelberg.de \\ 3 Main Astronomical Observatory, 27 Academica Zabolotnogo Str., 03680 Kiev, Ukraine \\ e-mail: nkhar@mao.kiev.ua \\ ${ }^{4}$ Institute of Astronomy of the Russian Acad. Sci., 48 Pyatnitskaya Str., Moscow 109017, Russia \\ e-mail: piskunov@inasan.rssi.ru
}

Received 21 January 2005 / Accepted 18 April 2005

\begin{abstract}
We present a list of 130 Galactic Open Clusters, found in the All-Sky Compiled Catalogue of 2.5 Million Stars (ASCC-2.5). For these clusters we determined a homogeneous set of astrophysical parameters such as size, membership, motion, distance and age. In a previous work, 520 already-known open clusters out of a sample of 1700 clusters from the literature were confirmed in the ASCC-2.5 using independent, objective methods. Using these methods the whole sky was systematically screened for new clusters. The newly detected clusters show the same distribution over the sky as the known ones. It is found that without the a priori knowledge about existing clusters our search lead to clusters which are, on average, brighter, have more members and cover larger angular radii than the 520 previously-known ones.
\end{abstract}

Key words. techniques: photometric - catalogs - astrometry - stars: kinematics - open clusters and associations: general Galaxy: stellar content

\section{Introduction}

For many years the major sources of open cluster lists were based on visual inspection of photographic plates. The presentday highly homogeneous and accurate all-sky surveys like the Hipparcos and Tycho catalogues (ESA 1997), or the 2MASS near-IR survey (Cutri et al. 2003) gave new impetus to a systematic search for new clusters. Platais et al. (1998) profited from the use of Hipparcos proper motions and parallaxes and detected six nearby associations and nine candidate open clusters. Using the photometric and kinematical data of the Tycho- 2 catalogue (Høg et al. 2000), Alessi et al. (2003) detected 11 new clusters and determined their ages, geometric and kinematical parameters. Dutra et al. (2003) and Bica et al. (2003) searched the 2MASS for compact embedded clusters in the direction of known nebulae. The visual inspection of $J, H, K_{\mathrm{s}}$ images lead to the discovery of 346 infrared clusters, stellar groups and candidates all over the Milky Way. All new optical clusters and candidates are listed in a catalogue by

$\star$ Tables 2 and 3 are only available in electronic form at http://www. edpsciences.org

$\star \star$ The complete set of data files for all 130 clusters is only available in electronic form at the CDS via anonymous $\mathrm{ftp}$ to cdsarc.u-strasbg.fr (130.79.128.5) or via

http://cdsweb.u-strasbg.fr/cgi-bin/qcat?J/A+A/440/403
Dias et al. (2002). These authors also maintain an online list of catalogues (DLAM hereafter) ${ }^{1}$, which is updated at regular intervals.

Our work is based on a catalogue of 2.5 million stars with proper motions in the Hipparcos system and $B, V$ magnitudes in the Johnson photometric system, spectral types (ASCC-2.5; Kharchenko 2001) and radial velocities, if available in the Catalogue of radial velocities of galactic stars with high precision astrometric data (Kharchenko et al. 2004a). The ASCC- 2.5 can be retrieved from the $\mathrm{CDS}^{2}$; a detailed description of the catalogue can be found in Kharchenko (2001) or in the corresponding ReadMe file at the CDS. In a previous paper (Kharchenko et al. 2004b, hereafter Paper I), we used the ASCC-2.5 to identify known open clusters and compact associations, and developed an iterative pipeline for the construction of cluster membership based on combined spatial/kinematical/photometric criteria. For 520 known clusters a uniform set of structural (location, size), kinematical (proper motions and radial velocities) and evolutionary (age) parameters was derived (Kharchenko et al. 2005, referred hereafter as Paper II). The results encouraged us to start a search for new clusters in the ASCC-2.5.

\footnotetext{
${ }^{1}$ http://www.astro.iag.usp.br/ wilton/.

2 ftp://cdsarc.u-strasbg.fr/pub/cats/I/280A/.
} 


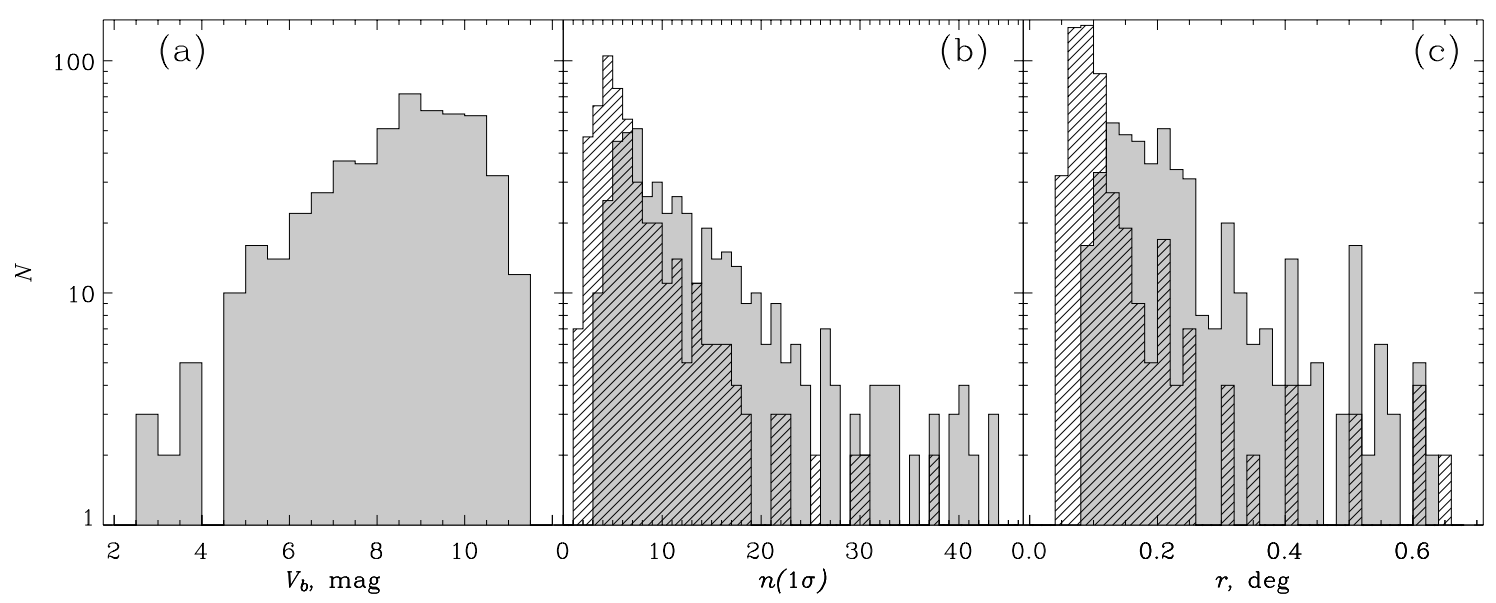

Fig. 1. Distribution of the COCD clusters over magnitude of the brightest star among the most probable cluster members a), over number of most probable members in a cluster b), over angular radius of a cluster $\mathbf{c}$ ). The hatched histograms in b) and c) are for core radii, whereas the filled histograms are related to corona radii. For convenience, long tails in the distributions in b) and c) are truncated.

The present paper describes this systematic search for new open clusters. During this search for new clusters in the catalogue ASCC-2.5, we discovered 130 clusters. 21 of them are listed in the online list DLAM as private communications. This paper is the first presentation of these 21 clusters in a refereed publication. Instead of a visual inspection of sky surveys, we implement a multi-factor search pipeline, which is based on the analysis of properties of known clusters already identified in the ASCC-2.5. As a result we could increase our sample of clusters present in the ASCC- 2.5 by about $20 \%$, determine memberships and derive a uniform set of basic astrophysical parameters in the same way as for the 520 previously known clusters.

The paper has the following structure. In Sect. 2 we discuss the properties of known clusters identified in the ASCC-2.5. These properties give us useful hints for the search of new clusters. In Sect. 3 we present details of the search procedure applied. In Sect. 4 we describe the sample of the newly discovered clusters and compare their properties with those of known clusters already identified in the ASCC-2.5. In Sect. 5 we summarise the results.

\section{Properties of known open clusters identified in the ASCC-2.5}

For each star, the ASCC-2.5 gives the equatorial coordinates, proper motions, $B$, and $V$ magnitudes. Only for a minority of them are spectral and luminosity classes and radial velocities also known. Therefore, starting from the data content of the ASCC-2.5, we suggest and adopt the following strategy for searching for new open clusters. This strategy is based on a clustering analysis in the multi-dimensional space of equatorial coordinates and proper motions with a follow-up check of colour-magnitude distributions of the candidates. Since the completeness and especially the accuracy of the ASCC-2.5 data show a strong dependence on stellar magnitude, a straightforward search routine must take these correlations into account. Furthermore, a successful approach requires a set of starting parameters that are related to typical properties of the given survey (e.g. mean surface density of stars, the limiting magnitude, wavelength range). The choice of starting parameters should yield a reasonable relation between the number of cluster candidates selected at the beginning and the number of real clusters confirmed at the end.

In this work we made use of the experience obtained from the identification of known open clusters in the ASCC-2.5. In that study we could find 520 of some 1700 known clusters (Paper I), and we derived cluster parameters such as sizes, distances, ages and space velocities (Paper II). The resulting parameters and supplementa information on these clusters were gathered in the Catalogue of Open Cluster Data (COCD) supplemented by the Open Cluster Diagrams Atlas (OCDA). The main reason that "only" $30 \%$ of known open clusters were confirmed with the ASCC-2.5 data is the relatively bright limiting magnitude $\left(V_{\lim } \approx 12\right)$ of the catalogue. Also, a number of clusters from the 1700 known ones did not pass at least one of the criteria based on spatial, kinematical or photometric $(B$, $V$ ) data. Considering the distribution of appropriate parameters of the confirmed clusters, we can define selection criteria that will help to improve the chance of detecting new clusters in the ASCC-2.5. This approach also gives a statistical basis for the strategy of searching.

The evident selection parameters among the number of cluster properties are those related to the population and structure of a cluster as an enhanced density of brighter stars following a cluster main sequence. These properties can be translated to lower level descriptors like the number of bright cluster members located within a specified area around the cluster centre.

In Fig. 1 the distributions of 520 clusters from the COCD (see Paper II) are shown as functions of three relevant cluster parameters: the magnitude of the brightest star among the most probable cluster members, the number of the most probable members in a cluster and the angular size of a cluster. The most probable members are defined in Paper I as stars for which the individual proper motions, magnitudes, and colours deviate from the mean proper motion and the "isochrone" of the cluster by less than one $\sigma$ rms (" $1 \sigma$-members" i.e., stars with 
Table 1. Threshold values of clustering descriptors adopted in this study.

\begin{tabular}{lccccc}
\hline \hline Descriptor & $\hat{V}_{\mathrm{s}}$ & $\hat{r}_{\mathrm{s}}$ & $\hat{r}_{\mathrm{c}}$ & $\hat{n}_{\mathrm{s}}$ & $\hat{n}_{\mathrm{c}}$ \\
Value & $9.5 \mathrm{mag}$ & $0.3 \mathrm{deg}$ & $0.15 \mathrm{deg}$ & 8 & 5 \\
\hline
\end{tabular}

membership probabilities $P \geq 61 \%$ ). From Fig. 1 we may conclude that a typical cluster from the COCD has more than seven $1 \sigma$-members with at least one star brighter than $V=9$. Inner cluster regions have a higher stellar surface density; they are called cores in Paper II and typically include five $1 \sigma$-members. Although for a few clusters in the COCD, the core and corona radii reach sizes of 3 and 6 degrees, respectively, for the vast majority $(70-80 \%)$ of the clusters the members are concentrated within 0.15 deg (i.e. a typical core radius), but some members are found up to $0.30 \mathrm{deg}$ (i.e. a typical corona radius) from the cluster centre.

\section{Description of the search procedure}

Our search procedure begins with the selection of "seeds", i.e. bright stars with magnitudes $V \leq \hat{V}_{\mathrm{s}}$ where $\hat{V}_{\mathrm{s}}$ is the adopted limiting magnitude. At this step, we consider each seed as if it were the most probable kinematical member in the centre of a cluster. Then, we select all ASCC-2.5 stars located at distances less than the adopted cluster radius $\hat{r}_{\mathrm{s}}$. The derived sample is checked for a clustering in the multi-dimensional space of coordinates and proper motions. If a clustering is revealed, we apply our general pipeline of selecting cluster members in a celestial field and determine cluster parameters as described in Papers I and II. The pipeline is run twice. For candidates of clusters, the first run provides initial estimates of geometric parameters and distances from the Sun. Also, it removes those candidates for which we are not able to derive these parameters (e.g. due to false clustering, or a lack of necessary data). The second run removes co-moving (non-member) stars and provides the final list of cluster members as well as the complete set of cluster parameters.

The crucial point of the search strategy is the selection of the threshold parameters which provide optimum starting conditions for a decision of whether or not a real clustering exists. A common proper motion differing significantly from the field would be a good criterion. In general, we should assume that unknown clusters would have relatively small proper motions (otherwise, they would already have been found). By increasing $\hat{V}_{\mathrm{s}}$ and $\hat{r}_{\mathrm{s}}$, we would find more cluster candidates, but the number of clusters confirmed at the end would grow slowly and finally stop. From preliminary tests we found that a reasonable "cost-to-performance relation" can be achieved with threshold parameters based on the statistics given in Sect. 2 .

The search procedure uses the descriptors and their thresholds as listed in Table 1 . The quantity $\hat{r}_{\mathrm{s}}$ is the maximum search radius (analogous to the cluster radius). In order to take into account the expected negative gradient of stellar density in a cluster, we introduce a core radius $\hat{r}_{\mathrm{c}}$. The minimum numbers of members within the cluster area and core are called $\hat{n}_{\mathrm{s}}$ and $\hat{n}_{\mathrm{c}}$, respectively. The proper motions of members must follow the proper motion of the corresponding seed (i.e. analogous to $1 \sigma$-members).

The detailed procedure for searching for new clusters consists of the following steps:

1. Selection of seed stars. All ASCC- 2.5 stars with $V<\hat{V}_{\mathrm{s}}$ and $B-V<2$ mag which are not $1 \sigma$-members of known clusters are considered as seeds. Altogether, about 221000 stars have been selected for further tests.

2. Construction of cluster candidates. In a circle centred on a seed with radius $\hat{r}_{\mathrm{s}}$, we select all ASCC-2.5 stars whose proper motions are known with a mean error $\varepsilon_{\mathrm{pm}}<$ 10 mas/yr. Following the definitions in Paper I and assuming the corresponding seed star "s" to be a cluster member and its proper motion $\mu_{x}^{\mathrm{s}}, \mu_{y}^{\mathrm{s}}$ to represent the mean cluster proper motion, we compute the kinematical probability $P_{\mathrm{i}}^{\mathrm{s}}$ of the cluster membership for each star "i i" in the circle as

$P_{\mathrm{i}}^{\mathrm{s}}=\exp \left\{-\frac{1}{4}\left[\left(\frac{\mu_{x}^{\mathrm{i}}-\mu_{x}^{\mathrm{s}}}{\varepsilon_{\mu_{x}^{\mathrm{i}}}+\delta \varepsilon}\right)^{2}+\left(\frac{\mu_{y}^{\mathrm{i}}-\mu_{y}^{\mathrm{s}}}{\varepsilon_{\mu_{y}^{\mathrm{i}}}+\delta \varepsilon}\right)^{2}\right]\right\}$,

here $\mu_{x}$ and $\mu_{y}$ correspond to $\mu_{\alpha} \cos \delta$ and $\mu_{\delta}$, respectively. $\delta \varepsilon$ is a correction for external errors of the proper motions in the ASCC-2.5 (see Paper I). A sub-sample of stars with $P_{\mathrm{i}}^{\mathrm{s}}>61 \%$ (" $1 \sigma$-members") is separated. If this sub-sample consists of $n_{\mathrm{s}} \geqslant \hat{n}_{\mathrm{s}}$ stars with at least $\hat{n}_{\mathrm{c}}$ stars within the inner circle of a radius $\hat{r}_{\mathrm{c}}$, we include this area in the following tests. After this second step we obtained 4767 sky fields containing cluster candidates. Some of them are overlapping areas.

3. Elimination of overlapping areas. If neighbouring cluster candidates have several stars in common, only those with the largest number $\hat{n}_{\mathrm{s}}$ have been considered. Cases of double and triple overlaps were treated automatically, whereas a few cases of quadruple overlapping were treated manually. After this step only 2472 candidate areas were retained for the next step.

4. Preliminary selection of cluster members and determination of cluster parameters. This step and the following step 5 are based on the pipeline developed in Papers I and II for member selection and determination of cluster parameters. Now we consider a larger sky region of $2 \times 2$ square degrees around each remaining seed star. Additionally to the nominal kinematical selection, we carry out a simplified photometric selection, which removes stars located below the Main Sequence. Thus, at this stage, we possibly keep some red field stars which, by chance, show the same proper motions as a given cluster candidate. As expected, the vast majority of cluster candidates does not show any Main Sequence and was excluded from further considerations. We are also forced to remove those cluster candidates that do not contain at least one "kinematical and photometric member" with known spectral classification. In the current study, the spectral classification is the only information given in the ASCC-2.5 that can be used to derive estimates of distance and color excess. In a few cases, significant parallaxes (from Hipparcos) were available for the brightest probable cluster members. These parallaxes were used to check the derived distances. 
After this step, our list includes 308 cluster candidates with preliminary determined cluster memberships and with a number of preliminary cluster parameters such as the position of the cluster's centre, distance, and average proper motion.

5. Final determination of membership and cluster parameters. At this stage, the standard pipeline is applied with complete kinematical and photometric selection. After several iterations for each cluster, co-moving red stars are excluded and the final cluster membership is established. The complete set of cluster parameters including the cluster age is derived. If cluster members are present in the ASCC-2.5 subsample of stars with radial velocities (Kharchenko et al. 2004a), the mean radial velocity of the cluster is computed. Only 130 clusters have passed this stage. The other 178 candidates have been excluded since after rejection of co-moving red stars, no more clustering in the space of coordinates and proper motions could be observed.

6. Visual inspection with sky maps. In a final step, supplementing the search for new clusters, we inspected Digitised Sky Survey $(\mathrm{DSS})^{3}$ and SuperCOSMOS Sky Survey (SSS) ${ }^{4}$ blue and red images. The size of an image around a given cluster was selected according to the determined cluster corona radius with a minimum size of $30 \times 30 \mathrm{arcmin}^{2}$, and up to $180 \times 180 \mathrm{arcmin}^{2}$ for the largest clusters. The results of the visual inspection of the fields with new clusters are included in the notes to the extension of the OCDA. For about 30 cluster areas the notes are with respect to the presence of nebulae and/or varying surface density of faint stars, which could not yet been mentioned in the ASCC-2.5 sky maps.

\section{Results of the search and comparison with properties of known clusters}

We present the 130 new clusters in Tables 2 and $3^{5}$. Among them we found 21 in the 15/Feb./2004-update of DLAM (see Table 3). They were not included in the COCD with its 520 clusters, because the COCD had already been finished before this update became available. In DLAM only cluster centres and angular sizes are given for these 21 clusters, which were privately communicated to the authors of the data base. We detected these clusters, however, without using DLAM data as preliminary input and determined astrophysical parameters for them. Therefore, we consider them as independently confirmed. The celestial positions of these 21 confirmed clusters are given in Table 3. On the other hand, about a dozen of the new clusters (also privately communicated) in that update of DLAM could not be confirmed in our work.

At the moment of submission of this paper, we did not find any published information on the other 109 clusters. Therefore,

\footnotetext{
${ }^{3}$ http://archive.stsci.edu/.

${ }^{4}$ http://www-wfau.roe.ac.uk/sss/pixel.html/.

5 Table 2 including only coordinates and sizes of 109 new clusters as well as Table 3 with coordinates and sizes of 21 confirmed clusters are available only in the electronic version of the Journal. The complete set of data files for all 130 clusters is only available in electronic form at the CDS.
}

we consider them as unknown clusters to date. They are listed in Table 2.

The current data refer to 130 sky areas and have the same content and format as the CSOCA described in Paper I. This file, which we call "The 1st Extension of CSOCA", includes 26778 stars, with 10161 of them located within the determined cluster radii. According to the combined spatial/kinematical/photometric criteria, 6203 stars are classified as cluster members and 2127 out of them as $1 \sigma$-members. For all these clusters, a homogeneous set of basic astrophysical parameters is derived and the corresponding cluster diagrams are prepared. Again, the data are presented in the same way as for the 520 known clusters (see Paper II) and called "The 1st Extension of the COCD" and "The 1st Extension of the OCDA", respectively. An example of a page in the Extension Atlas is shown in Fig. 2 for the open cluster ASCC 13. The complete set of data files, extending the CSOCA, COCD and OCDA, is available in electronic form only via the $\mathrm{CDS}^{6}$.

In Fig. 3 we compare two cluster samples retrieved from the ASCC-2.5. One can see that new clusters show the same distribution over the sky as the already known objects (Fig. 3a). Also, we may conclude that the applied search strategy probably puts some limitation on the detection of new clusters: the clusters from the COCD Extension are, on average, brighter (Fig. 3b), they have more members (Fig. 3c), and they cover larger areas (Fig. 3d) than the known clusters from the main COCD catalogue. Whereas one new cluster is found at a distance of $5 \mathrm{kpc}$, the bulk is located within $1 \mathrm{kpc}$ from the Sun. The range of ages is comparable for both samples, although a higher fraction of young $(\log t \approx 7)$ clusters is observed in the COCD Extension.

\section{Summary and outlook}

Starting from 221000 stars in ASCC-2.5 which have passed the test for seeds of possible open clusters, we applied our search procedure based on spatial, kinematical and photometric criteria, and at the end of the study, we found 130 new open clusters. For each of these clusters a complete set of relevant parameters (memberships, locations, sizes, distances, ages, proper motions, and - for 69 clusters - radial velocities) was derived. It seems to be a paradox, but we now have more basic information on these new clusters than on many others already reported as known clusters for a long time. In our search for new clusters we profited from all-sky astrometric and photometric surveys which became available in recent years. In Papers I and II we used the ASCC-2.5 to identify known open clusters, to redefine (or to confirm) the membership, and to derive a uniform set of astrophysical parameters for these clusters. The preliminary information already published for these clusters was very helpful for this study, too. In our search for new clusters, however, without any a priori information, we needed at first clear criteria to decide whether an apparent clustering was indeed a real physical open cluster.

Comparing the histograms in Figs. 3b,c for the clusters from the COCD and from the COCD Extension, we can con-

\footnotetext{
${ }^{6}$ http://vizier.u-strasbg.fr/cgi-bin/VizieR/.
} 


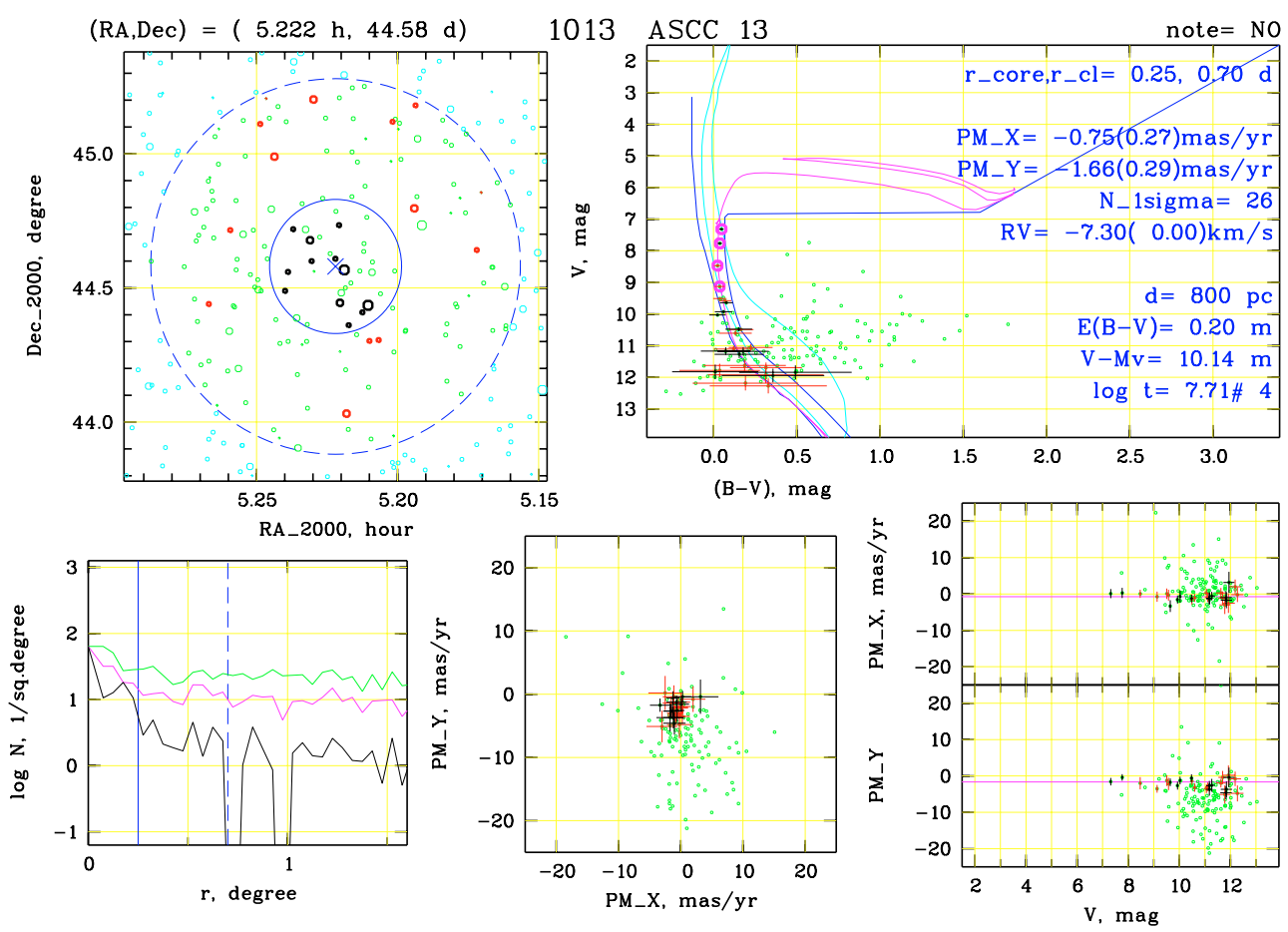

Fig. 2. Example of the spatial, kinematical and evolutionary parameters of the new open cluster ASCC 13. Left upper panel is a sky map of the cluster neighbourhood. The small circles are stars, their size indicates stellar magnitude (only in this panel). In all other diagrams stars are shown as grey dots. The error bars indicate the rms-errors in the corresponding data for $1 \sigma$-members. The large circles in the sky map outline the cluster core (solid) and corona (dashed). The cross indicates the cluster center. The upper right panel is the CMD of the cluster. Bold circles show the stars used for the calculation of the average age of the cluster. The curves are: the empirical ZAMS (leftmost), the red edge of the MS (thick line to the right), the isochrone corresponding to the calculated age, and the limits of the evolved MS (light curves). The legend within the CMD displays the derived parameters of the cluster. The lower left panel shows radial profiles of the projected density. The curves (from the top to the bottom) correspond to all stars, all members, $1 \sigma$-members. Vertical lines mark the core (solid) and cluster radii (dashed), respectively. The middle panel is the vector point diagram of proper motions. The two rightmost panels are "magnitude equation" $\left(\mu_{x, y}-V\right.$ relation) diagrams, showing the proper motion of cluster members as a function of magnitude. The horizontal lines show the average proper motion of the cluster.



Fig. 3. Open clusters identified with ASCC-2.5 data: the comparison of previously known (COCD) and newly discovered (COCD Extension) clusters. Panel a) shows the distribution of clusters over the sky. The crosses indicate known clusters, the circles are for newly discovered ones. Histograms b)-f) are normalised to the number of known clusters $\left(N_{\text {tot }}=520\right)$, the filled and hatched histograms are for known and newly discovered clusters, respectively. Panel b) is the distribution of clusters over the magnitude $V_{\mathrm{b}}$ of the brightest $1 \sigma$-member. Panel c) shows the distribution over the number of $1 \sigma$-members in a cluster, whereas panels d)-f) are the distributions of clusters over angular radius, distance and age, respectively. For convenience, long tails in c)-e) are truncated. 
clude that the newly discovered clusters are, on average, more prominent objects. There is possibly a potential to find poorer clusters in the ASCC-2.5 by diminishing the threshold values in Table 1. In this case, we must take into account a considerable increase of candidates with a lower success rate for a final confirmation as undoubted clusters.

Assuming a similar distribution of $V_{\mathrm{b}}$ and $n(1 \sigma)$ for the known and for the new clusters, we suspect from (Figs. 3b,c) that the ASCC-2.5 should contain stars belonging to about 100 more still unknown open clusters. But without accurate data at faint magnitudes, without any knowledge of their distances, it will be difficult to confirm them. We were already forced to reject a number of "good" candidates in step 4 of the search procedure due to the lack of spectral classification. Therefore, a more successful approach has to wait until more accurate data at fainter magnitudes is available.

Note added in proof: Eric Mamajek (private communication) draw our attention to the fact that ASCC-16 was previously mentioned as a clustering of stars by Briceño et al. (2005).

Acknowledgements. This work was supported by the DFG grant 436 RUS 113/757/0-1, the RFBR grant 03-02-04028, and by the FCNTP "Astronomy". We acknowledge the use of the Simbad database and the VizieR Catalogue Service operated at the CDS, France, and of the WEBDA facility at the Observatory of Geneva, Switzeland.

\section{References}

Alessi, B. S., Moitinho, A., \& Dias, W. S. 2003, A\&A, 410, 565

Bica, E., Dutra, C. M., Soares, J., \& Barbuy, B. 2003, A\&A, 404, 223

Briceño, C., Calvet, N., Hernández, J., et al. 2005, AJ, 129, 907

Cutri, R. M., et al. 2003, in University of Massachusetts and Infrared Processing and Analysis Center, IPAC/California Institute of Technology, VizieR On-line Data Catalog: II/246

Dias, W. S., Alessi, B. S., Moitinho, A., \& Lépine, J. R. D. 2002, A\&A, 389, 871

Dutra, C. M., Bica, E., Soares, J., \& Barbuy, B. 2003, A\&A, 400, 533

ESA: 1997, Hipparcos and Tycho catalogues, ESA-SP 1200, Cat. I/239

Høg, E., Fabricius, C., Makarov, V. V., et al. 2000, The Tycho-2 Catalogue: Positions, proper motions and two-colour photometry of the 2.5 million brightest stars, Copenhagen, CD-ROM distribution

Kharchenko, N. V. 2001, Kinemat. Phys. Celest. Bodies, 17, 409

Kharchenko, N. V., Piskunov, A. E., \& Scholz, R.-D. 2004a, Astron. Nachr., 325, 439

Kharchenko, N. V., Piskunov, A. E., Röser, S., Schilbach, E., \& Scholz, R.-D. 2004b, Astron. Nachr., 325, 743 (Paper I)

Kharchenko, N. V., Piskunov, A. E., Röser, S., Schilbach, E., \& Scholz, R.-D. 2005, A\&A, 438, 1163 (Paper II)

Platais, I., Kozhurina-Platais, V., \& van Leeuwen, F. 1998, AJ, 116, 2423 
N. V. Kharchenko et al.: 109 new Galactic open clusters, Online Material p 1

\section{Online Material}


N. V. Kharchenko et al.: 109 new Galactic open clusters, Online Material p 2

Table 2. List of 109 newly-discovered clusters (cluster coordinates $\alpha_{\mathrm{c}}$ in hours, $\delta_{\mathrm{c}}$ in degrees, respectively for J2000). The cluster radii $r_{\mathrm{cl}}$ (in degrees) are also given. Note that the complete set of cluster parameters is available only in electronic form via the CDS (see Sect. 4).

\begin{tabular}{|c|c|c|c|c|c|c|c|c|c|c|c|}
\hline Cluster & $\alpha_{\mathrm{c}}$ & $\delta_{\mathrm{c}}$ & $r_{\mathrm{cl}}$ & Cluster & $\alpha_{\mathrm{c}}$ & $\delta_{\mathrm{c}}$ & $r_{\mathrm{cl}}$ & Cluster & $\alpha_{\mathrm{c}}$ & $\delta_{\mathrm{c}}$ & $r_{\mathrm{cl}}$ \\
\hline ASCC 1 & 0.160 & 62.68 & 0.20 & ASCC 43 & 7.885 & -28.17 & 0.35 & ASCC 85 & 16.792 & -45.46 & 0.08 \\
\hline ASCC 2 & 0.331 & 55.71 & 0.30 & ASCC 45 & 8.264 & -35.65 & 0.20 & ASCC 87 & 17.050 & -28.45 & 0.15 \\
\hline ASCC 3 & 0.519 & 55.28 & 0.21 & ASCC 46 & 8.276 & -48.51 & 0.40 & ASCC 88 & 17.113 & -35.60 & 0.30 \\
\hline ASCC 4 & 0.886 & 61.58 & 0.40 & ASCC 48 & 8.575 & -37.61 & 0.32 & ASCC 90 & 17.652 & -34.80 & 0.15 \\
\hline ASCC 5 & 0.966 & 55.84 & 0.13 & ASCC 51 & 9.300 & -69.69 & 0.66 & ASCC 91 & 17.815 & -37.36 & 0.10 \\
\hline ASCC 6 & 1.787 & 57.73 & 0.30 & ASCC 52 & 9.466 & -54.26 & 0.27 & ASCC 93 & 18.137 & -22.26 & 0.08 \\
\hline ASCC 7 & 1.982 & 58.97 & 0.25 & ASCC 53 & 9.632 & -59.55 & 0.31 & ASCC 94 & 18.260 & -14.99 & 0.10 \\
\hline ASCC 8 & 2.347 & 59.61 & 0.30 & ASCC 54 & 9.746 & -54.44 & 0.22 & ASCC 95 & 18.268 & -25.71 & 0.10 \\
\hline ASCC 9 & 2.782 & 57.73 & 0.17 & ASCC 55 & 9.905 & -57.08 & 0.23 & ASCC 98 & 18.710 & -33.63 & 0.20 \\
\hline ASCC 11 & 3.538 & 44.84 & 0.35 & ASCC 56 & 10.137 & -64.37 & 0.35 & ASCC 99 & 18.818 & -18.73 & 0.10 \\
\hline ASCC 12 & 4.832 & 41.73 & 0.25 & ASCC 57 & 10.180 & -66.68 & 0.38 & ASCC 100 & 19.027 & 33.57 & 0.14 \\
\hline ASCC 13 & 5.222 & 44.58 & 0.70 & ASCC 58 & 10.252 & -54.97 & 0.40 & ASCC 101 & 19.227 & 36.33 & 0.15 \\
\hline ASCC 14 & 5.342 & 35.22 & 0.22 & ASCC 59 & 10.337 & -57.65 & 0.35 & ASCC 102 & 19.414 & 29.95 & 0.08 \\
\hline ASCC 15 & 5.377 & 36.55 & 0.20 & & & -58.48 & 0.12 & & & 18.69 & 0.15 \\
\hline ASCC 16 & 5.410 & 1.80 & 0.62 & ASCC 61 & 10.769 & -56.86 & 0.32 & ASCC 105 & 19.696 & 27.38 & 0.20 \\
\hline ASCC 17 & 5.420 & 30.17 & 0.25 & ASCC 62 & 10.848 & -60.10 & 0.28 & ASCC 107 & 19.809 & 21.96 & 0.07 \\
\hline ASCC 18 & 5.436 & 0.82 & 0.62 & ASCC 63 & 10.931 & -60.41 & 0.15 & ASCC 108 & 19.897 & 39.37 & 0.08 \\
\hline ASCC 19 & 5.463 & -1.98 & 0.80 & ASCC 64 & 11.051 & -60.92 & 0.18 & ASCC 109 & 19.900 & 34.58 & 0.15 \\
\hline ASCC 20 & 5.479 & 1.63 & 0.75 & ASCC 65 & 11.185 & -61.12 & 0.22 & ASCC 110 & 20.050 & 33.57 & 0.10 \\
\hline ASCC 21 & 5.483 & 3.65 & 0.80 & ASCC 66 & 11.227 & -55.42 & 0.30 & ASCC 111 & 20.187 & 37.45 & 0.20 \\
\hline ASCC 23 & 6.339 & 46.67 & 0.36 & ASCC 67 & 11.692 & -61.02 & 0.20 & ASCC 113 & 21.200 & 38.60 & 0.12 \\
\hline ASCC 24 & 6.479 & -7.02 & 0.35 & ASCC 69 & 12.110 & -69.77 & 0.40 & ASCC 114 & 21.667 & 53.97 & 0.08 \\
\hline ASCC 25 & 6.759 & 24.60 & 0.21 & ASCC 70 & 12.250 & -64.43 & 0.30 & ASCC 115 & 21.948 & 51.48 & 0.08 \\
\hline ASCC 26 & 6.840 & 7.25 & 0.17 & ASCC 71 & 12.345 & -67.52 & 0.41 & ASCC 116 & 21.976 & 54.49 & 0.08 \\
\hline ASCC 27 & 6.898 & -4.39 & 0.20 & ASCC 72 & 12.550 & -60.95 & 0.25 & ASCC 117 & 22.083 & 62.27 & 0.11 \\
\hline ASCC 28 & 6.901 & -0.17 & 0.30 & ASCC 73 & 12.610 & -67.29 & 0.40 & ASCC 119 & 22.320 & 46.90 & 0.08 \\
\hline ASCC 29 & 6.905 & -1.65 & 0.22 & ASCC 74 & 13.597 & -58.81 & 0.20 & ASCC 120 & 22.510 & 57.21 & 0.10 \\
\hline ASCC 30 & 6.950 & -6.21 & 0.26 & ASCC 75 & 13.786 & -62.42 & 0.17 & ASCC 121 & 22.512 & 54.90 & 0.10 \\
\hline ASCC 31 & 7.015 & 3.50 & 0.17 & ASCC 76 & 13.871 & -66.40 & 0.35 & ASCC 122 & 22.554 & 39.61 & 0.25 \\
\hline ASCC 33 & 7.053 & 25.05 & 0.90 & ASCC 77 & 14.180 & -62.33 & 0.32 & ASCC 123 & 22.710 & 54.26 & 0.28 \\
\hline ASCC 34 & 7.175 & 6.07 & 0.30 & ASCC 78 & 15.085 & -68.39 & 0.16 & ASCC 125 & 22.938 & 62.75 & 0.30 \\
\hline ASCC 35 & 7.211 & 2.12 & 0.40 & ASCC 79 & 15.320 & -60.73 & 0.52 & ASCC 126 & 23.105 & 51.05 & 0.10 \\
\hline ASCC 36 & 7.242 & -21.12 & 0.18 & ASCC 80 & 15.410 & -60.14 & 0.25 & ASCC 127 & 23.140 & 64.85 & 0.18 \\
\hline ASCC 37 & 7.301 & -24.48 & 0.16 & ASCC 81 & 15.782 & -50.98 & 0.26 & ASCC 128 & 23.343 & 54.60 & 0.15 \\
\hline ASCC 38 & 7.453 & -5.55 & 0.26 & ASCC 82 & 15.790 & -64.41 & 0.30 & ASCC 130 & 23.880 & 62.44 & 0.08 \\
\hline ASCC 39 & 7.550 & -22.95 & 0.30 & ASCC 83 & 15.837 & -52.80 & 0.21 & & & & \\
\hline ASCC 40 & 7.560 & -13.76 & 0.18 & ASCC 84 & 15.915 & -60.74 & 0.25 & & & & \\
\hline
\end{tabular}


N. V. Kharchenko et al.: 109 new Galactic open clusters, Online Material p 3

Table 3. List of 21 confirmed clusters (cluster coordinates $\alpha_{\mathrm{c}}$ in hours, $\delta_{\mathrm{c}}$ in degrees, respectively for J2000). Cluster radii $r_{\mathrm{cl}}$ are in degrees. Previous names and radii of the cluster candidates (i.e. the only parameters which were provided by DLAM) are given in brackets. Note that the full astrophysical parameter set determined in the present paper is only available in electronic form via the CDS (see text).

\begin{tabular}{lrrc}
\hline \hline Cluster & $\alpha_{\mathrm{c}}$ & $\delta_{\mathrm{c}}$ & $r_{\mathrm{cl}}$ \\
\hline ASCC 10 (Alessi-Teutsch 9) & 3.450 & 35.04 & $0.48(0.36)$ \\
ASCC 22 (Ferrero 11) & 6.242 & 0.64 & $0.18(0.11)$ \\
ASCC 32 (Alessi 33) & 7.033 & -26.50 & $0.50(0.54)$ \\
ASCC 41 (Herschel 1) & 7.784 & 0.02 & $0.36(0.10)$ \\
ASCC 42 (Alessi-Teutsch 3) & 7.881 & -53.01 & $0.36(0.29)$ \\
ASCC 44 (Alessi 34) & 8.028 & -50.57 & $0.40(0.48)$ \\
ASCC 47 (Alessi-Teutsch 7) & 8.530 & -39.08 & $0.50(0.24)$ \\
ASCC 49 (Teutsch 38) & 8.798 & -37.99 & $0.45(0.37)$ \\
ASCC 50 (Alessi 43) & 8.838 & -41.72 & $0.40(0.38)$ \\
ASCC 68 (Alessi-Teutsch 8) & 12.049 & -60.92 & $0.20(0.12)$ \\
ASCC 86 (Alessi-Teutsch 12) & 17.033 & -59.01 & $0.55(0.30)$ \\
ASCC 89 (Alessi 24) & 17.388 & -62.64 & $1.10(0.75)$ \\
ASCC 92 (Alessi 31) & 17.852 & -11.88 & $0.30(0.22)$ \\
ASCC 96 (Ferrero 1) & 18.335 & -32.37 & $0.25(0.12)$ \\
ASCC 97 (Alessi 40) & 18.616 & -19.22 & $0.43(0.40)$ \\
ASCC 103 (Teutsch 35) & 19.603 & 35.67 & $0.36(0.18)$ \\
ASCC 106 (Alessi 44) & 19.719 & 1.60 & $0.66(0.50)$ \\
ASCC 112 (Alessi-Teutsch 11) & 20.274 & 52.10 & $0.22(0.20)$ \\
ASCC 118 (Alessi-Teutsch 5) & 22.140 & 61.10 & $0.21(0.18)$ \\
ASCC 124 (Alessi 37) & 22.802 & 46.25 & $0.30(0.24)$ \\
ASCC 129 (Alessi J2327+55) & 23.467 & 55.60 & $0.21(0.21)$ \\
\hline
\end{tabular}

